\title{
Der Stechlin: Fontanes neues Sprachbewußtsein
}

Das in den letzten Jahren so auffällig angestiegene Interesse der Literaturwissenschaft an Theodor Fontanes Romanwerk hat - glücklicherweise - den Autor nicht kanonisiert. Man stößt in der neueren Fontaneliteratur auf die widersprechendsten Urteile:

Für Martini hat Unwiederbringlich gegenüber den früheren Werken des Autors »an erzählerischer Kraft eingebüßt «; ${ }^{1}$ Demetz hält dieses Buch für ein Meisterwerk, weil es nicht politisch ist, weil es ein "ganz mit dem Silberstift entworfene[r] Roman ohne historischen Ballast und politische Bürde ${ }^{2}$ ist; Günther hält es umgekehrt für ein Meisterwerk, weil es politisch ist, weil darin »gleichnishaft [...] in der verfehlten Restitution des Privaten die Unmöglichkeit der Restitution im Staatlichen vorweggespiegelt $\aleph^{3}$ ist. - Killy wählt Cécile für die Fischer Taschenbuchreihe Exempla classica aus, die aus 100 »Meisterwerken der abendländischen Literatur « bestehen soll; nach Nürnberger zeigt dieser Roman Fontane »am Beginn der Meisterschaft «, ${ }^{4}$ Brinkmann findet, daß gerade dieses Werk - und L'Adultera, »die schwächsten Glieder in der Reihe der Berliner Romane sind «; ${ }^{5}$ als Romane, mit denen Fontane »der ererbten, aber leblosen Methodik des Gesellschaftsromans « ${ }^{6}$ vertraut, nennt Demetz Cécile und Graf Petöfy; während Reuter nun wieder von dem »seinen künstlerischen Qualitäten nach noch immer unterschätzten « ${ }^{7}$ Graf Petöfy spricht. - Demetz zählt Fontanes Kriminalgeschichten »zu seinen geringeren Produktionen $;_{;}^{8}$ prompt antwortet Thomas mit einer Ehrenrettung von Unterm Birnbaum. ${ }^{9}$ - Demetz urteilt über Effi Briest, das Buch sei »nicht ganz frei von plötzlicher Sentimentalität $«,{ }^{10}$ und er erwähnt dabei besonders »die Sentimentalität der Schlußkapitel $;^{11}$ er zählt aber den geheimnisvollen Chinesen zu den »dichterischen Gestaltungen, die mit mechanischer Begriffsumgrenzung keineswegs zu deuten sind ${ }^{12},{ }^{2}$ auf der anderen Seite meint Stern, der Chinese sei »the only blemish « des Romans, alles übrige aber, »and it is virtually die whole work - theme, agents, and narrative manners - achieve a rare degree of integration ${ }^{13}{ }^{13}$

Diese Skala von Widersprüchen, die wieder einmal über die mangelnde objektiv-wissenschaftliche Basis literaturwissenschaftlicher Urteile zu denken gibt, macht deutlich, welche große Rolle bei den Interpreten das jeweilige Vorverständnis von Literatur spielt. Jedenfalls scheint es in dieser Situation der Fontaneforschung angebracht zu sein, die Texte selbst so genau wie möglich zu lesen, um eine empirische Grundlage für wertende Urteile zu gewinnen. Man wird das umso unbesorgter tun können, als bei aller Divergenz der 
Auffassungen eins doch unterdessen klar geworden zu sein scheint: Wenn es überhaupt einen deutschen Romancier im 19. Jahrhundert gibt, der den aus kulturellen, sozialen und politischen Gründen bedingten Provinzialismus der deutschen Literatur dieser Zeit überwindet und sich im Kreise der Balzac, Stendhal und Flaubert, Dickens, Melville und James, Tolstoi und Dostojewkij behaupten kann, dann ist es Theodor Fontane.

Die Forschungslage im Hinblick auf Fontanes letzten Roman Der Stechlin, dem ich mich widmen möchte, um einige, wie mir scheint, noch nicht genügend beachtete Züge dieses Buches zu diskutieren, ist von der oben beschriebenen nicht verschieden. Noch immer zerfallen seine Interpreten - grob gesagt - in 2 Gruppen. Die eine hält dieses Werk für den Gipfel Fontanescher Erzählkunst, für »einen deutschen Roman von weltliterarischem Rang «, ${ }^{14}$ für ein zukunftsweisendes Romanwerk; die andere - extrem formuliert - für das charmante und subtile, aber uferlose Geplauder eines anekdotenliebenden Greises, der gegenüber Frau Jenny Treibel und Effi Briest doch schon entschieden an künstlerisch formender Kraft verloren hat. Ich verhehle von Anfang an nicht, daß ich der ersten Gruppe zuneige, und meine, daß schon das Messen dieses Romans an früheren Werken Fontanes ein Fehler sein könnte, wenn Der Stechlin etwas anderes als sie intendiert. Die Abwertung dieses Buches drückt ein Vermissungserlebnis aus: In ihm steht nicht das Handlungsmäßige im Vordergrund, und alles andere hat dienende Funktion, und so spricht man von mangelnder Konzentration des Textes. Auch Demetz in seinem Fontanebuch fällt diesen Verkennungen zum Opfer, wenn er in einer Reihe von Dort-Hier-Vergleichen schreibt: dort, in den früheren Romanen Fontanes, Dialektik, Dramatik und Funktionalität, Handlungsfortgang »in einer federnden Spirale [..], die rhythmisch und stoßweise in die Höhe steigt $\ll ;{ }^{15}$ hier, im Stechlin, Addition, Biographie, Sich Lösen von Teilen »aus dem funktionalen Zusammenhang der Erzählung, ${ }^{16}$ gestalterische Mittel, die nur mit »der strengsten Disziplin ${ }^{17}{ }^{7}$ zu einer kompositorischen Einheit führen könnten, die eben fehlt. Die Folge nach Demetz: "Die Wucherung beginnt. ${ }^{18}$ Käme es aber nicht doch darauf an, das Neue am Stechlin nicht als bloßen Abfall vom Alten zu beschreiben - Demetz benutzt bezeichnenderweise Gegenbegriffe von an früheren Romanen gewonnenen Termini - sondern die eigene Gesetzlichkeit und neuartige Disziplin, den neuen Typ von kompositorischer Einheit im Stechlin zu entdecken?

$\mathrm{Da} ß$ seine beiden letzten Romane von den vorhergehenden strukturell verschieden sind, hat Fontane sehr wohl gewußt und sogar als Fortschritt seiner Schriftstellerei empfunden. Er schreibt über Die Poggenpuhls: »Das Buch ist 
kein Roman und hat keinen Inhalt. Das $>$ Wie $<$ muß für das $>$ Was $<$ eintreten mir kann nichts Lieberes gesagt werden. $\ll{ }^{19} » \mathrm{Daß}$ man dies Nichts, das es ist, um seiner Form willen so liebenswürdig anerkennt, erfüllt mich mit großen Hoffnungen $[\ldots] \cdot \ll^{20}$ Fontanes ausführlichste Aussage zum Stechlin lautet:

der Stoff, so weit von einem solchen die Rede sein kann - denn es ist eigentlich bloß eine Idee, die sich einkleidet - dieser Stoff wird sehr wahrscheinlich mit einer Art Sicherheit Ihre Zustimmung erfahren. Aber die Geschichte, das was da erzählt wird. Die Mache! Zum Schluß stirbt ein Alter und zwei Junge heiraten sich; - das ist so ziemlich alles, was auf 500 Seiten geschieht. Von Verwicklungen und Lösungen, von Herzenskonflikten oder Konflikten überhaupt, von Spannungen und Überraschungen findet sich nichts. [...] Alles Plauderei, Dialog, in dem sich die Charaktere geben, und mit ihnen die Geschichte. Natürlich halte ich dies nicht nur für die richtige, sondern sogar für die gebotene Art, einen Zeitroman zu schreiben $[\ldots] .^{21}$

Für beide Romane besteht also Fontane ausdrücklich auf dem, was er tut; er hält es nicht nur für gelungen, sondern im Sinne literarischer Entwicklungen für notwendig. Wenden wir uns wieder ausschließlich dem Stechlin zu. Fontane betont in dem zitierten Briefausschnitt dreierlei: Das Werk hat keine Handlung; es ist vielmehr eine Idee, die sich einkleidet; und das ist die gebotene Art, einen Zeitroman zu schreiben. Diese Aussagen evozieren Fragen: Was ist die Idee, die sich einkleidet; was, wenn nicht eine sinnträchtige Handlung, ist der >poetische Reif Zeitroman durch diesen neuen Typus?

Bei dem Versuch, diese Fragen zu beantworten, kann vor allem ein Forschungsansatz von Nutzen sein, nämlich »die Preisgabe der Fabel und die beherrschende Rolle der Gespräche « als »eine Konsequenz der eigentlichen Vorstellung vom Wesen des Zeitromans « $^{22} z u$ interpretieren, wie es Wolfgang Preisendanz in dem Fontanekapitel seines Buches Humor als dichterische Einbildungskraft getan hat. Er kommt zu folgendem Ergebnis: Das Spezifische des Fontaneschen Humors in Der Stechlin ist die Darstellung der großen "geschichtsbildenden Kräfte und Bewegungen « »im durchschnittlichen und privaten Leben der Menschen $«{ }^{23}$ Es $»$ ist von recht umfassenden Aspekten der Wirklichkeit die Rede, aber diese Aspekte werden an sehr partikulären oder bagatellmäßigen Details vergegenwärtigt; das Nächste wird dem Fernsten verbunden, das scheinbar Kontingente steht für Zusammenhänge, die Spannung zwischen Allgemeinem und Besonderem, Gemeintem und Bezeichnetem ist extrem «. ${ }^{24}$ Preisendanz zeigt vor allem an den Anfangskapiteln des Romans, »wie alle [...] Gespräche zu Spiegeln einer Totalbestimmung des Menschlichen werden, d. h. wie sie unabhängig von Anlaß und Gegenstand 
manifestieren, daß alles Menschliche in Zusammenhang, in Kommunikation mit der aktuellen Geschichtszeit steht. Denn gerade, wenn die privaten, alltäglichen, banalen und trivialen Momente der menschlichen Wirklichkeit als Gesprächsanlässe oder -gegenstände plötzlich die Dimension des Aktuellen aufweisen, wenn sie plötzlich bezeugen, wie alles Menschliche im wesentlichen Bezug zur Gesamtsituation steht und diesen Bezug gleichsam impliziert, erweist sich die Zeitbedingtheit des Menschen als Totalbestimmung «. ${ }^{25}$

Diese auf sorgfältige Beobachtung aufgebaute Deutung erklärt wohl die neue Intention von Fontanes letztem Roman, sie beleuchtet aber nicht, wie ein solcher auf das Menschliche als Totalbestimmung gerichteter Roman ohne den Zusammenhalt einer sich entwickelnden Handlung textliche Integration erreichen, wie aus dem Zeitroman ein Zeitroman werden konnte, woran die literarische Qualität des Werkes zu hängen scheint. Folgt man Preisendanz aber so weit, dann bietet sich leichter die Möglichkeit, die neuen, dem neuen Ziel von Der Stechlin angemessenen Gestaltungsmittel zu finden, die seine literarische Einheit herstellen.

Demnach intendiert dieses Buch >epische Totalität<. Das Thema des Werkes ist nicht handlungsbestimmt oder biographisch. Es wäre falsch zu erwarten, daß sein Autor alle Elemente auf die konsequente Zeichnung von Charakteren im Sinne einer sich dialektisch entwickelnden und sich steigernden Handlung konzentriert, die an einem durch die Fabel gegebenen Punkt endet. Das Ende ist der natürliche, nicht der dramatische Tod wie in Schach von Wuthenow, Graf Petöfy, Cécile, Stine, Unwiederbringlich und Effi Briest. Das Buch versucht vielmehr, „das Allgemeine, Prinzipielle, die Tendenzen und Zusammenhänge der Zeit in eine konkrete Vorstellung zu versammeln ${ }^{26}{ }^{26}$ Alles Dargestellte also, die Gespräche, Milieus, Schilderungen von Personen und Vorgängen sind nicht Funktionsträger im Hinblick auf eine Handlung, sondern im Hinblick auf eine "Idee «: auf das Menschliche, wie es sich in einer bestimmten Zeit als historisch Variables und Konstantes ausdruickt, im Hinblick auf den Zeitgeist<. Und damit ist das bisherige Abhängigkeitsverhältnis in Fontanes Romanen verschoben. Das Zeitbild, die religiöse, politische, soziale, künstlerische und psychologische Wirklichkeit, das gesellschaftliche und menschliche Verhalten, bisher anwesend im erzählten Fall, emanzipiert sich und wird selbst zum Thema des Buches. Der Roman ist nicht mehr wie bisher eine individuelle Geschichte, verwoben mit dem Zeitbild, sondern es ist dieses Zeitbild selbst, wie es, perspektivisch gebrochen durch die Personen, erscheint.

Die Welt in nuce, wie sie der geheimnisvolle See Stechlin enthält, den Fontane ausdrücklich als $» d a s$ Thema $\aleph^{27}$ des Buches bezeichnet hat, die preuBische Provinz als Mikrokosmos ohne eigentlich romanhaftes Geschehen, ohne »Verwicklungen und Lösungen, [...] Spannungen und Überraschungen «, wie Fontane in dem oben zitierten Brief gesagt hatte, konnte zwangsläufig 
nicht allein mit denselben literarischen Mitteln zu einer Romaneinheit gefügt werden wie die bisherigen handlungsbetonten Romane. In ihnen hatte Fontane ein Höchstmaß an Funktionalität und Zusammenhalt dadurch geschaffen, daß er auf eine im Laufe seiner Entwicklung als Romanautor immer beherrschtere und psychologisch begründetere Weise »das Dingliche zu indirekt sprechenden Sinnzeichen ${ }^{28}{ }^{28}$ verwandelte. Die erzählte Welt hatte Hinweischarakter für die Romanhandlung. Sie signalisierte dem Leser Bedeutungen in Abbreviaturen. Auch Der Stechlin ist noch voll von ihnen. Man denke nur an die kranke Aloe auf der Stechliner Schloßrampe mit ihrer vieldeutigen Symbolik, durch die unter anderem Dubslaw Rex schmunzelnd als einen Menschen entlarvt, der auf den bloßen Schein hereinfällt. ${ }^{29} \mathrm{Da}$ solchen Sinnzeichen aber die symbolische Funktion für die »Idee« versagt ist, wenn sie nicht über die Romanhandlung wirken, können sie nicht mehr allein die Einheit des Werks konstituieren. Diese muß durch ein Bezugssystem unterstützt werden, das dem Leser die universalia in re, die gelebten Gesetzlichkeiten des menschlichen Lebens als Thema des Buches signalisiert.

So machte Fontane nicht nur das Dingliche zu indirekt sprechenden Sinnzeichen, sondern vor allem das Sprachliche zu direkt sprechenden Sinnzeichen und erreichte damit eine neue Stufe seiner Romankunst. Er entwickelt noch in seinem letzten Roman ein neues Sprachbewußtsein. Er arbeitet im Stechlin zum ersten und einzigen Mal mit sprachlichen Grundmustern ${ }^{30}$ und ist damit auf dem Wege zum Roman des 20. Jahrhunderts. Ihn hat Rang folgendermaßen gekennzeichnet: »Als Schreibender will der Erzähler nicht mehr wissen, als was aus dem Sprechakt, dem Sprachprozeß von selbst hervorgeht. Der Text als Textur, als Wort- und Satzgewebe. ${ }^{31}$ Die 1. Hälfte dieses Satzes würde, auf Fontane angewendet, diesen überdeuten, die zweite indessen trifft auf den Stechlin zu. Es gibt in ihm, wie ich zu zeigen versuchen möchte, Wortfelder, variierte Sprachmuster und reflektierte Sprache, durch die die Gespräche als dominierender Bestandteil aufeinander bezogen werden. Und in dieser Verwendung von Schlüsselwörtern blickt Fontane über die Jahrhundertwende hinaus.

Das eigentliche Gerüst, das den Stechlin trägt und das in jeder Szene gegenwärtig ist, sind 3 sprachliche Komplexe, die kulturanthroplogische Kategorien bezeichnen, formale Prinzipien, die in jeweils einer zeitlich konkreten Ausfaltung dem individuellen menschlichen Leben den Rahmen geben, in dem der einzelne Mensch sich zu seiner Zeit verhält. Diese Schlüsselbegriffe besagen: Der Mensch steht in einer Spannung von alt und neu also in der geschichtlichen Zeit, in der Spannung von eng und weit, also im geographischen Raum, und in der Spannung von hoch und niedrig, also auf einer sozialen Stufenleiter. Es sind diese 3 Komplexe, die das in einem Zusammenhang stehende Netz bilden, innerhalb dessen sich Fontanes Gestalten in Der Stechlin zur geschichtlichen Situation: Preußen um 1900 verhalten. Man könnte dabei 
durchaus an Rothackers kulturanthropologische Kategorien denken, die er 1940 einführte, um eine kulturelle Situation im Wandel begrifflich zu erfassen. ${ }^{32}$

Wenn Demetz über den Stechlin schreibt: »Die Energie ist gemindert, die das Ganze zusammenhält $\ll,{ }^{33}$ kann er eigentlich nur meinen, daß Fontane die Handlung mit vielfältigen Beziehungen nicht ausarbeitet, denn die Energie, die bis ins kleinste Detail die »Idee« des Buches immer neu spiegelt und in Beziehungen setzt, ist geradezu erstaunlich. Nur kommt es dem Autor nicht mehr darauf an, menschliches Schicksal in Handlung darzustellen, sondern menschliches Sich Verhalten in einer geschichtlichen, geographischen und sozialen Lage. Die imaginären Zentren des Romans sind dazu die oben genannten kulturanthropologischen Kategorien. Wie verschieden auch immer die Gesprächsanlässe, die Themen, die Schauplätze oder die Personen sein mögen, sie alle beleuchten Einstellungen zu historischem Wandel, zu Leben in Heimat und Welt, zu sozialer Stellung in der Gesellschaft, und es sind sprachliche Reizwörter, die diese Zusammenhänge herstellen. Um der Konzentration willen und weil das Thema alt-neu bisher in der Fontaneliteratur die relativ größte Aufmerksamkeit gefunden hat, beschränke ich mich zur Illustration meiner These vor allem auf das Wortpaar Enge-Weite und seine Aspekte und versuche an einzelnen Stellen Durchblicke auf weitere Zusammenhänge des Buches zu geben.

Zur Illustration soll als Beispiel das erste Diner bei Dubslaw dienen, das das 3. Kapitel des 1. Teils einnimmt (24-36): Die Unterhaltungen bei Tisch scheinen sich mit den verschiedensten Themen zu beschäftigen, aber sie sind allesamt Variationen zu den 3 oben genannten Wortkomplexen und zeigen immer wieder neue Überlagerungen und Zusammenhänge zwischen ihnen.

Vergangenheit und Gegenwart sind gleich zu Anfang gegenwärtig in Dubslaws und Gundermanns Dialog über die neumodische Einführung der Telegraphie, wobei Gundermann sich als sozial aufgestiegener Bourgeois enthüllt, aber das Gespräch zieht gleich darauf auch den Aspekt Enge-Weite des Lebens mit ein, denn Dubslaw erwähnt die durch die Telegraphie gegebene Möglichkeit, mit jedem beliebigen Punkt der Erde Kontakt aufzunehmen (»so könnten wir den Kaiser von China wissen lassen, daß wir hier versammelt sind $\ll$ ).

Kurz darauf nimmt Czako noch einmal von dem Karpfen, weil »Fischnahrung jetzt obenan« (27) steht wegen ihres Phosphorgehalts. Frau Gundermann geht auf den Fortschritt der Naturwissenschaft, der darin angedeutet wird, nicht ein, sondern spricht über die neuen schwedischen Zündhölzer, die 
es früher nicht gab, auch dadurch wieder Stellung nehmend zum Wandel des Lebens. Czako fährt fort, über die Karpfen zu sprechen, und benutzt ihr sagenhaftes Alter, um auf das Geheimnis des Stechliner Sees zu kommen, wobei er die Fähigkeit des Sees, auf Weltereignisse, die einen Wandel bewirken, durch Eruptionen zu reagieren, zu einem Vergleich des Karpfens mit dem Bourgeois benutzt, der sich bei revolutionären Veränderungen verkriecht, bis die Gefahr vorüber ist. Und damit zeigt sich im Buch zum erstenmal, daß der See gerade mit denjenigen Themen verknüpft ist, die seine »Idee« darstellen, die das Gerüst des Werkes bilden. Das wird vor allem deutlich in dem Gespräch Melusines und Lorenzens, bei dem die erstere einige Schlüsselsatze des ganzen Romans spricht:

Ich respektiere das Gegebene. Daneben aber freilich auch das Werdende, denn eben dies Werdende wird über kurz oder lang abermals ein Gegebenes sein. Alles Alte, soweit es Anspruch darauf hat, sollen wir lieben, aber das Neue sollen wir recht eigentlich leben. (270)

Solche Sätze sprechen das Urteil über die Domina, die ausschließlich das Alte lebt. Melusine fährt fort:

Und vor allem sollen wir, wie der Stechlin uns lehrt, den großen Zusammenhang der Dinge nie vergessen. Sich abschließen, heißt sich einmauern, und sich einmauern ist Tod. Es kommt darauf an, daß wir gerade das beständig gegenwärtig haben . [...] [Woldemar] ist auch nicht geistig bedeutend genug, um sich gegen abweichende Meinungen, gegen Irrtümer und Standesvorurteile wehren zu können. (270 f.)

Alle 3 Themen sind hier im Zusammenhang mit dem See genannt. Bezeichnenderweise wird er aber auch noch offensichtlicher, wenn auch ironischerweise, mit dem Thema der sozialen Standesunterschiede in Beziehung gebracht. Woldemar spricht von den »vornehmen, geheimnisvollen Beziehungen« (135) des Stechlin, als Melusine sich erkundigt, was es mit ihm auf sich habe. Er erläutert dann: »Er steht mit den höchsten und allerhöchsten Herrschaften, deren genealogischer Kalender noch über den Gothaischen hinauswächst, auf du und du.« (136)

Auf der allerletzten Seite des Buches geschieht es sogar, daß der Erzähler diese Funktion des Sees, und damit seine eigene Wendung vom Handlungsroman zum Zustandsroman versteckt ausspricht. Der Brief, den Melusine an Lorenzen schreibt, mit dem sie ja schon einmal über den See gesprochen hat, spricht auch Fontanes neue Überzeugung aus: "Es ist nicht nötig, daß die Stechline weiterleben, aber es lebe der Stechlin.« (388) Das ist umso auffälliger, als die Gräfin damit eine Wandlung in ihren Anschauungen aus- 
drückt, denn noch in der Mitte des Buches, als sie zum erstenmal von dem See hörte, hatte sie gesagt: »De[r] >Stechlin<? Was ist das? [...] ich bin so glücklich zu wissen, daß es Stechline gibt. Aber der Stechlin! Was ist der Stechlin?« (135)

Kehren wir zurück zu Dubslaws Diner: Daß das Gespräch am anderen Ende der Tafel »sehr, sehr anders ging « (28), ist nur oberflächlich richtig und sicher auch Versteckspiel Fontanes. In Wirklichkeit bestehen auch hier die Verbindungsfäden weiter. Rex fragt Katzler, ob nicht sein früheres Leben, das ihn »in die weite Welt hinausgeführt « (29) habe, interessanter gewesen sei. Katzler ist durchaus zufrieden, und später, wenn der Leser den Superintendenten Koseleger kennenlernt, merkt er, daß beide Gestalten als Gegenbilder konstruiert sind, denn Koseleger ist derjenige, der aus der vornehmen und weiten Welt seiner Jugend nicht zurückfinden kann in den begrenzten Aufgabenbereich in der Mark - Vergangenheit und Gegenwart verbindet sich mit Welt und Heimat. Rex' Frage bildet den Anlaß für Überlegungen über Weite und Enge des persönlichen Lebensraums, wobei einige diesbezügliche Stichworte fallen, die das ganze Buch durchziehen im Zusammenhang mit diesem Themenkreis und die eben den Typ von Reizwort bilden, den ich oben zu charakterisieren versucht habe: $»$ Leben draußen in der Welt « oder in »unserer stillen Grafschaft«, »Auch-draußen-zu-Hause-Sein«, »Weltfremde«, »Einsamkeit und Stille«, »im Engen und Begrenzten«, »Enge des Daseins«, »jener stillen Stelle« (29 f.).

Fast alle diese Wörter tauchen an späteren Stellen des Buches wieder auf. Ich gebe ein paar Beispiele. Melusine schreibt an Woldemar: »In unserer sogenannten großen Welt gibt es so wenig, was sich zu sehen und hören verlohnt; das meiste hat sich in die stillen Winkel der Erde zurückgezogen.« (136) Lorenzen preist seinen Lieblingsautor mit den Worten: »Dieser Joao de Deus [...] war genau das, was ich wohl sein möchte, wonach ich suche, seit ich zu leben, wirklich zu leben angefangen, und wovon es beständig draußen in der Welt heißt, es gäbe dergleichen nicht mehr.« (158) Vom Grafen Barby heißt es: „Er hieß seine Gäste herzlich willkommen, weil er jederzeit das Bedürfnis hatte, von dem, was draußen in der Welt vorging, etwas zu hören.« (229) Dubslaw - Lorenzen teilt es Woldemar mit - nennt sich »weltfremd « (163) und bemerkt zu dem Polizisten Uncke: »Nu ja, in der Welt draußen da klappt nich immer alles. Aber so im Schoß der Familie [...].« (265)

Dubslaw ist es auch, der erzählt, während seiner Soldatenzeit habe er »Fühlung mit der großen Welt«(305) gehabt. Lorenzen erläutert Melusine die Epochen der preußischen Geschichte und meint, als »das ungeniale Land« plötzlich Genies hervorgebracht habe, habe man »doch mehr draußen in der Welt als daheim « (272) gestaunt. Es paßt zur Domina, daß sie sagt: "Mir widersteht das Fremde.« (283) Zum Schluß des Gesprächs bei Dubslaws Essen 
verbindet Lorenzen das Thema von Enge und Weite mit dem von alter und neuer Zeit:

»Innere Mission in nächster Nähe, sei's mit dem Alten, sei's mit etwas Neuem.«

"Also mit dem Neuen«, sagte Woldemar und reichte seinem alten Lehrer die Hand.

Aber dieser antwortete: »Nicht so ganz unbedingt mit dem Neuen. Lieber mit dem Alten, soweit es irgend geht, und mit dem Neuen nur, soweit es muß.«(31)

Unterdessen unterhalten sich Frau Gundermann und Czako über Berlinisches, und Frau Gundermann entblößt dabei ungewollt ihre kleinbürgerliche Herkunft und ihre Unbildung. Sie mißversteht fast jede an sie gerichtete Bemerkung und zeigt sich auch als gesellschaftlich ungeschliffen ("Frau von Gundermann schien auf das ihr als einziger, also auch ältester Dame zustehende Tafelaufhebungsrecht verzichten zu wollen und wartete $[\ldots] \ll, 36$ ). Der Leser allerdings, durch Andeutungen über die abwesende Frau Katzler und ihre hohe Herkunft neugierig gemacht, ahnt schon hier, was später immer deutlicher wird: Auch Frau Gundermann als diejenige, die in das Stechliner Milieu aufgestiegen ist, und die Prinzessin, die dazu abgestiegen ist, sind parallel konstruierte Gestalten, die das Thema der ständischen Ordnung und ihrer Verschiebungen beleuchten und die Verkrampfungen sozialer Nichtzugehörigkeit spüren lassen. Frau Gundermanns gesellschaftliche Geschmacklosigkeiten und Frau Katzlers fixe Idee der Pflichterfüllung sind in gleicher Weise menschliches Fehlverhalten, hervorgerufen durch die Fremdheit des Milieus, in dem sie leben. Von Lorenzen wird es an späterer Stelle, nämlich wieder in dem so wichtigen Gespräch mit Melusine, grundsätzlich ausgesprochen:

"Der Hauptgegensatz alles Modernen gegen das Alte besteht darin, daß die Menschen nicht mehr durch ihre Geburt auf den von ihnen einzunehmenden Platz gestellt werden. Sie haben jetzt die. Freiheit, ihre Fähigkeiten nach allen Seiten hin und auf jedem Gebiete zu betätigen. Früher war man dreihundert Jahre lang ein Schloßherr oder ein Leineweber; jetzt kann jeder Leineweber eines Tages ein Schloßherr sein.« »Und beinah auch umgekehrt«, lachte Melusine. (271)

Die Konversation zwischen Frau Gundermann und Czako ist die Unterhaltung zweier Emporkömmlinge, und Dubslaw belächelt die für eine Tischgesellschaft recht ungewöhnliche Thematik des Gesprächs: Ratten und die Pariser Katakomben. Czako, der an späterer Stelle, die noch zu zitieren sein 
wird, gesteht, daß er eigentlich keinerlei gesellschaftliche Erziehung genossen hat, repräsentiert in diesem Gespräch eine Art pervertierter Weltläufigkeit, die in Paris die Ratten und die Katakomben am interessantesten findet; und nur eine so unkultivierte Frau wie Frau Gundermann, die nebenbei zugleich auch ihren beschränkten Berliner Lokalpatriotismus im Sinne des Themas Enge-Weite zu erkennen gibt, kann das goutieren. Dubslaw fügt eine Bemerkung über Rom hinzu, und wiederum klingt damit ein Aspekt des Themas Enge-Weite an, der das ganze Buch durchzieht. Nicht nur die verschiedenen deutschen Länder kommen darin mit ihren Unterschieden gesprächsweise vor, besonders auffällig in dem Brief der Domina an Woldemar (160-162), sondern auch fast alle wichtigen Länder der Erde bilden Anlaß zu Erörterungen über Provinzialismus und Weltläufigkeit: Italien, Rußland, Frankreich, England, Amerika usw. Die Provinzialismen legen sich gewissermaßen in Ringen um die Menschen. Für Frau Gundermann ist schon Stechlin >Ausland<, und auch Torgelow, der neue sozialdemokratische Abgeordnete, wird als Fremder empfunden, weil ihn »euch die Berliner hergeschickt haben« (202). Solche Unterschiede zwischen Provinz und Stadt, die beide aus weiterer Sicht wiederum zusammen als Provinz größerer Ordnung erscheinen, werden etwa apostrophiert, wenn Czako sagt: »Die Betten auf dem Land sind überhaupt das beste (46), oder wenn Dubslaw einwirft: »Schon mein Vater sagte mal: $\mathrm{Ja}$, wir auf dem Lande, wir haben immer noch die alten Wiener Kongreßwitze.«(52)

Woldemar sagt zu seiner Tante, mancher wolle »in seinem Potzdam lieber der Erste, als in seinem Berlin der Zweite sein « (101). Er selbst bekommt durch seine Englandreise (»Ja, reisen und in den Krieg ziehen, da lernt man, da wird man anders «, 338) den notwendigen Abstand, der es ihm nach der Rückkehr ermöglicht, die richtige Entscheidung zwischen Melusine und Armgard zu treffen. Er kehrt am Ende des Buches nach Stechlin zurück, weil er erfahren hat: »Die Scholle daheim, die dir Freiheit gibt, ist doch das Beste.« (387) Sogar von den sozialdemokratischen Globsowern wird er »als einer der >Ihrigen $<$ begrüß « (388).

Nur von solchen thematischen, nicht handlungsbedingten Zusammenhängen her sind auch die Nebenpersonen des Romans zu erklären, deren Funktion einzusehen einigen Interpreten so schwer fällt. Frau Schickedanz etwa, die Besitzerin des Hauses, in dem die Barbys wohnen, ist der Typ der großstädtischen Berlinerin, die doch aus mangelnder Welterfahrung provinziell bleibt:

Wie beinah jedem hierlandes Geborenen, war auch ihr die Gabe wirklichen Vergleichenkönnens völlig versagt, weil jeder echte, mit Spreewasser getaufte Berliner [...] seinen Zustand nur an seiner eigenen kleinen Vergangenheit, nie aber an der Welt draußen mißt, von der er, wenn 
er ganz echt ist, weder eine Vorstellung hat, noch überhaupt haben will. [...] Sie rangierte sich ein und wies sich, soweit ihre historische Kenntnis das zuließ, einen ganz bestimmten Platz an: Fürst Dolgorucki, Herzog von Devonshire, Schickedanz. (121 f.)

Bemerkenswert ist, daß hier mit der Frage des Lebensraums gleich wieder die soziale Stellung verbunden ist. Es ist zweifellos kein Zufall, daß Fontane die Barbys gerade bei dieser Frau wohnen läßt, denn sie stehen auf der genau entgegengesetzten Seite. Der alte Graf, schreibt Woldemar in sein Tagebuch, "weiß - was sie hierzulande nicht wissen oder nicht wissen wollen -, daß hinter dem Berg auch noch Leute wohnen. Und mitunter noch ganz andre«. (117)

Die andern hinter dem Berg sind immer auch anwesend im Gespräch. Man hat gewisse Vorlieben für oder Abneigungen gegen ganze Länder: Die Domina liebt nur die Mark; Woldemar schätzt alles Skandinavische; Wrschowitz haßt es; Melusine läßt »Irland [...] fallen« und nimmt »lieber Indien, woher aller gute Geschmack kommt « (112). Auch solche geographischen und politischen Sympathien sind der Mode unterworfen, also wieder mit dem Thema alt-neu verknüpft. Dubslaw etwa ist altmodisch wegen seiner prorussischen Haltung. Wer >up to date < ist, schwärmt auch nicht mehr für das Französische, sondern für Englisches. Adelheid - natürlich - ist gegen beides: "Sage nichts Französisches, das verdrießt mich immer. Manche sagen jetzt auch Englisches, was mir noch weniger gefällt.«(100)

Kehren wir zu einer letzten Bemerkung zu Dubslaws Diner zurück. Auch der letzte Gesprächsfetzen dabei bezieht sich noch auf das Thema Enge-Weite und hat nur als Beitrag zu diesem Thema überhaupt eine Funktion. Der 24 jährige Sohn der Gundermanns, meint Dubslaw, »müßte mehr raus. Er versauert hier ein bißchen», und als Frau Gundermann antwortet: »Er will nicht fort. Er sagt, zu Hause sei es am besten« (36), stimmt Dubslaw zu. Der Zusammenhang der ganzen Dinerszene beruht also, wie ich gezeigt zu haben hoffe, darauf, daß sich thematische Bezüge um die Begriffe alt-neu, eng-weit und hoch-niedrig bilden, nicht darauf, daß sich Handlung entwickelt. Um diesen Angelpunkt aber gegenwärtig zu halten, benutzt Fontane ein abstraktes Gerüst von Schlüsselworten als Integrationsprinzip des Romans. Bei der Konzentration, mit der die Themen auf subtilste Weise auf jeder Seite anwesend sind, zumal in den Gesprächen, deren Vorherrschaft im Stechlin man schon immer hervorgehoben hat, scheint es wenig angebracht, von nachlassender Formkraft bei Fontane zu sprechen. Es wäre leicht, an immer neuen Szenen des Buches zu zeigen, wie sie alle an unsichtbaren Fäden zusammenhängen. Um auch scheinbare »Wucherungen« in diesen Rahmen einzuordnen, seien noch 2 besonders fernliegende Beispiele kurz betrachtet.

Die scheinbar ganz unmotiviert auftretende Nichte des Barbyschen Portiers, die als Dienstmädchen ihre Stellung alle 4 Wochen wechselt, weil ent- 
weder die sozialen Bedingungen so schlecht sind oder der Hausherr ihr zu nahe tritt, ist nur dann richtig zu verstehen, wenn man sie im Gegensatz zu den alten treuen Dienern Engelke und Jeserich sieht. Beide Parteien vertreten den Gegensatz zwischen alt und neu im Bereich des Dienstpersonals. Obwohl die sozialen Unterschiede in der alten Zeit viel strenger und offensichtlicher waren, waren die menschlichen Beziehungen zwischen Herren und Dienern doch intensiver. Wie schon bei der Wahl des sozialdemokratischen Abgeordneten stellt sich auch hier heraus, daß der Fortschritt in sozialer Hinsicht lediglich die Verantwortlichkeit der Herrschenden beseitigt hat, so daß eine menschliche Beziehungslosigkeit eingetreten ist. In dem Maß, in dem die Verpflichtung der Herren abnimmt, wird die Aufgabe der Dienenden zum bloßen >job<.

Auch die umrätselte Geschichte von der siamesischen Prinzessin gehört durchaus in den großen Zusammenhang des Buches. Man hat wohl bisher zu wenig beachtet, daß ihr Ausgangspunkt die Eskapade eines adligen Mädchens mit einem bürgerlichen Hauslehrer ist. Als ihnen auf ihrer Flucht nach England das Geld ausgeht, kehrt sie in ihr Elternhaus zurück und ist bereit, ihren adligen Vetter zu heiraten. Die Frage, an der sich die von Thormeyer erzählte Geschichte entzündet, ist, ob der Vetter sie nach ihrer vorübergehenden Mesalliance noch heiraten kann. Die Entsühnung von der Mesalliance in der orientalischen Erzählung ist aus der alten Vorstellung heraus möglich, daß Blut sühne. Aber es enthüllt die Dekadenz des märkischen Adels, daß in seiner Vorstellung die Entsühnung des adligen Fräuleins besonders deshalb für möglich gehalten wird, weil es »sehr hübsch und nebenbei auch noch sehr reich « (197) ist. Wieder also sind in dieser Episode alle 3 Grundthemen des Buches anwesend: Die neue Zeit nimmt die Moral nicht mehr ernst, wenn es um die eigenen Vorteile geht, während in dem siamesischen Märchen alle menschlichen Reaktionen in den Traditionen der gelebten Kultur verwurzelt sind. Das Mädchen zieht die Heimat mit Geld, aber ohne Liebe der Fremde ohne Geld, aber mit Liebe vor. Die sozialen Verhältnisse sind in Unordnung geraten, denn eine Adlige flieht mit einem Bürgerlichen.

Jeder Versuch allerdings, Fontanes Roman lediglich als Beitrag zu kulturanthropologischen Theorien zu betrachten, muß ihn zerstören. 2 nähere $\mathrm{Be}$ stimmungen aber wird man noch geben können. Das Stichwortnetz der Kategorien fängt unendliche Möglichkeiten des menschlichen Verhaltens ein und widerstrebt gerade dadurch der Theorie, wenn man die beiden zusammengehörigen Begriffe, wie auch Rothacker es mit seinen kulturanthropologischen Determinanten tut, als Endpunkte einer Skala sieht, so daß sich zwischen alt und neu, eng und weit, hoch und niedrig beliebig viele Zwischenstufen ergeben.

Weiterhin müssen alle 3 Wortkombinationen im Hinblick auf das konkrete Menschenleben zusammengenommen werden. Sie sind verwoben zu einem 
Geflecht, und auf jeden Menschen trifft eine bestimmte Kombination aller drei zu. Die Domina etwa ist für Enge, für Adel und für das Alte, und entsprechend vereinigen sich in ihr diese Züge zu einer Mischung, die ihr Bruder als »geradezu petrefakt« (284) bezeichnet. Koseleger ist für Adel, für das Alte, aber im Gegensatz zu Adelheid für die Weite. Dubslaw ist überhaupt kein Prinzipienreiter. Er ist von Fall zu Fall für das eine oder andere und damit der Gegenpol seiner Schwester. Für ihn enthält die Wirklichkeit überhaupt nur Einzelfälle, die geprüft werden müssen. Vielleicht könnte man sagen, daß Lorenzen das theoretisch zu bedenken versucht, was Dubslaw lebt. Er ist für eine abgewogene Kombination der Elemente: Wirken im Engen, aber aufgrund von Weltverständnis und -kenntnis; für den sozialen Ausgleich, aber auch für Respekt vor den gewachsenen sozialen Strukturen, wenn ihre Vertreter dessen würdig sind; für das Neue, aber nur soweit es besser ist als das Alte. Auch er kann deshalb dem einzelnen Fall kein Schema überstülpen, sondern muß die Wirklichkeit kritisch von Fall zu Fall prüfen.

Bei solchen Schematisierungen müßte mindestens zum Verständnis der einzelnen Gestalt noch hinzugenommen werden, in welchem Maß sie das zu leben Gelegenheit hat, was sie vertritt oder wieweit es Traum bleiben muß, denn hier liegen in Fontanes Augen Quellen des persönlichen Glücks und Unglücks, und Allerlei Glück, die Fragment gebliebene Konzeption eines frühen Romans, der verschiedenen Glücksvorstellungen nachgehen sollte, ist ein lebenslanges Thema Fontanes geblieben: In fast jedem seiner Romane gibt es ein Gespräch über Glück. Im Stechlin ist etwa Koseleger unglücklich, weil er seine Neigung zur Lebensweite nicht verwirklichen kann.

Wenn Fontane davon spricht, daß seiner Meinung nach Der Stechlin den zeitgemäßen Typ des Zeitromans darstellt, dann scheint es nach der obigen Darlegung des sprachlichen Grundgerüstes dieses Buches klar zu sein, daß der Autor offenbar sah, daß er mit dem Abbau der dramatischen Handlung und der Betonung menschlicher Verhaltensvariationen den 'Zeitgeist< in viel konzentrierterer Form einfangen konnte. Sein neues Sprachbewußtsein - und das unterstützt die hier vorgetragene These weiter - zeigt sich noch auf einer anderen, hiermit im Zusammenhang stehenden Ebene, die, soweit ich sehe, von der Forschung noch fast gar nicht beachtet worden ist. ${ }^{34}$

Zum erstenmal ist jetzt in Fontanes Romanen die Sprache nicht nur Medium, sondern Objekt des Gesprächs. Die Personen im Stechlin zeichnen sich durch ein bisher bei Fontane unbekanntes kritisches Verhältnis zu ihrer eigenen Sprache aus. Sie sprechen nicht nur; sie reflektieren Sprache. Sie haben prinzipiell ein gebrochenes Verhältnis zu der Sprache, die sie benutzen. 
Auch hier soll ein herausgegriffenes Beispiel die These wieder illustrieren. Als die Barbyschen Damen zum erstenmal nach Stechlin kommen und man den Spaziergang zum See unternimmt, unterhält sich Dubslaw mit Gendarm Uncke. Es geht zunächst um Torgelows Auftreten im Reichstag. Er hat schon eine Rede gehalten, aber sie war kein Erfolg. Dubslaw, der diese Information von Uncke bezieht, sagt darauf:

»Er wird wohl die Sache noch nicht recht weghaben. Ich meine das, was sie jetzt das Parlamentarische nennen. Das schad't aber nichts und ist eigentlich egal. Wichtiger is, wie sie hier in unserm Ruppiner Winkel, in unserm Rheinsberg-Wutz über ihn denken. Sind sie denn da mit ihm zufrieden?«

"Auch nicht, Herr Major. Sie sagen, er sei zweideutig."

»Ja, Uncke, so heißt es überall. Das is nu mal so, das is nicht zu ändern. In Frankreich heißt es immer gleich >Verrat und hier sagen se >zweideutig<. Da war auch einer von uns, den ich nicht nennen will, von dem hieß es auch so ....

"Von dem hieß es auch so. Ja, Herr Major. Und Pyterke, der immer gut Bescheid weiß, der sagte mir schon damals in Rheinsberg: >Uncke, glauben Sie mir, da hat sich der Herr Major eine Schlange an seinem Busen großgezogen $\ll$ «

»Kann ich mir denken; klingt ganz nach Pyterke. Der spricht immer so gebildet. Aber is es auch richtig?«

»Is schon richtig, Herr Major. Herr Major denken immer das Gute von 'nem Menschen, weil Sie so viel zu Hause sitzen und selber so sind. Aber wer so rum kommt wie ich. Alle lügen sie. Was sie meinen, das sagen sie nich, und was sie sagen, das meinen sie nich. Is kein Verlaß mehr; alles zweideutig.«

»Ja, so rund raus, Uncke, das war früher, aber das geht jetzt nicht mehr. Man darf keinem so alles auf die Nase binden. Das is eben, was sie jetzt >politisches Leben< nennen.«

»Ach, Herr Major, das mein ich ja gar nicht. Das Politische ... Jott, wenn einer sich ins Politische zweideutig macht, na, dann muß ich ihn anzeigen, das is Dienst. Darum gräm ich mich aber nich. Aber was nich Dienst is, was man so bloß noch nebenbei sieht, das kann einen mitunter leid tun. So bloß als Mensch.« (264)

In diesem kurzen Gespräch von etwa einer Textseite wird fünfmal eine Bemerkung über Wörter und ihren richtigen oder falschen Gebrauch gemacht. Zunächst sagt Dubslaw, Torgelow beherrsche noch nicht das, "was sie jetzt das Parlamentarische nennen «, dann flicht er ein, daß man in Frankreich Thema Enge-Weite - leicht von »Verrat« (sicher eine Anspielung auf die 
Dreyfus-Affäre), in Preußen von »zweideutig« spreche, was Uncke wenig später Gelegenheit gibt, den Mißbrauch, die Falschheit der Wörter insgesamt als Zeichen der Zeit zu werten. Dubslaw analysiert zwischendurch Pyterkes bildhafte Wendung, er habe »sich eine Schlange an seinem Busen großgezogen«. Er konterkariert solche Prätentionen dadurch, daß er selbst ein weniger ambitiöses, volkstümlicheres Bild benutzt, wobei er wieder über Worte philosophiert. Jemandem nicht alles auf die Nase binden, sei, "was sie jetzt politisches Leben nennen«. Aber Uncke als preußischer Gendarm versteht die Kombination von politisch und zweideutig prompt falsch und spricht von »anzeigen«. Unckes Naivität wird noch an einer anderen Stelle des Romans lächerlich gemacht, wobei Dubslaw wieder über Worte nachdenkt:

»Aber jeder will heutzutage hoch hinaus. Das is, was sie jetzt die >Signatur der Zeit nennen. Haben Sie den Ausdruck schon gehört, Uncke?«

»Zu Befehl, Herr Major.« (364)

Es ist bezeichnend, daß sich die angeführten Bemerkungen über Sprache, die Dubslaw macht, wiederum genau auf die 3 Themenkreise beziehen, die den Mittelpunkt dieser Beobachtungen am Stechlin bilden. In ihnen wird Geschichtliches im Wandel, Soziales im Wandel oder Enge des Lebensraums greifbar. Kritische Bemerkungen über Wörter und Bilder, sprachliche Mißverständnisse - eine der Quellen von Fontanes Humor - durchziehen den gesamten Dialog. Die Gestalten verhalten sich im Gespräch also nicht nur zur Wirklichkeit, über die sie sprechen, sondern sie verhalten sich immer auch zur Sprache, mit der sie die Wirklichkeit bezeichnen.

Gegen diese Beobachtung wären an dieser Stelle zwei Einwände möglich. Der zitierte Dialog könnte eine Ausnahme sein, die keine Verallgemeinerung erlaubt. Tatsächlich aber enthält fast jedes Gespräch im Stechlin diese neue Dimension des Dialogs. Darüber hinaus könnte die sprachliche Reflexion ein Mittel sein, Dubslaw zu charakterisieren als denjenigen, der auch sprachlich den falschen Schein durchschaut. Aber auch dieser Einwand trifft nicht $\mathrm{zu}$, denn nicht nur Dubslaw, sondern fast alle Gestalten des Romans - Rex, Czako, Woldemar, Melusine, Adelheid - sprechen zugleich und diskutieren Sprache. Es handelt sich also um ein Prinzip der Gestaltung.

Verschiedene Gestalten sagen etwa im Laufe des Buches von einem einzelnen Satz, einer Wendung, einem Wort, sie sei »trivial« (Czako, 17), »anstöBig« (Woldemar, 18), »verdammt« (Czako, 21), »altmodisch« (Dubslaw, 55), »schrecklich« (Czako, 71), »plebeje« (Woldemar, 75), »plebeje und minderwertig« (Rex, 108), "etwas schwerfällig" (Thormeyer, 197), »furchtbar« (Melusine, 296), »zu doll« (Dubslaw, 326), »etwas fortgeschritten« (Dubslaw, 368). Es gibt die "neue Facon, in der die Berliner sprechen« (Adelheid, 98); es gibt »Lieblingswörter« (Dubslaw, 181) und »Lieblingswendungen« (Rex, 
29), Wörter aus der »Diebsprache« (Dubslaw, 357) und »feine Wendungen« (Dubslaw, 361). Zwar hatte Fontane seinen Gestalten schon immer eine bestimmte Sprache gegeben - man denke nur an die Verwendung des Plattdeutschen oder die feinfühlige Adaption von Zitaten ${ }^{35}$ - aber er hatte sie bisher nicht über Sprache philosophieren lassen. Jetzt ordnen sie selbst das gesprochene Wort in einen sozialen, historischen, geographischen oder psychologischen Kontext ein und legen damit auch sprachlich den überindividuellen Rahmen bloß, in den sie eingespannt sind. Auch hier scheint die Abnahme der dramatischen Handlung in einem Verhältnis zur Verwendung dieses neuen literarischen Mittels zu stehen, denn die sprachlichen Reflexionen führen uns die Gestalten vor, ohne daß sie handeln müssen. Es fällt dabei auf, daß an einigen Stellen des Buches Sprache grundsätzlich als Medium der Wahrheitsfindung, wenn auch ironisch, in Frage gestellt wird. Auf die Bemerkung in dem Gespräch mit Dubslaw habe ich schon verwiesen, aber in dem Roman fällt auch der Satz »Wörter sind Kinderklappern«. (238) Wenn es auch Cujacius ist, der ihn ausspricht, ein Mann, der durchaus nicht zu den Schweigsamen gehört.

Eine Szene zwischen den Geschwistern Stechlin enthält folgende Passage:

»Ja, Schwester, du hast gut reden. So sicher wie du wohnt eben nicht jeder. Adelheid! das ist ein Name, der paßt immer. Und im Kirchenbuche, wie mir Lorenzen erst neulich gezeigt hat, steht sogar Adelheide. Das Schluß->e < ist bei der schlechten Wirtschaft in unserem Hause so mit draufgegangen. Die Stechline haben immer alles verurscht.«

»Ich bitte dich, wähle doch andere Worte.«

»Warum? Verurscht ist ein ganz gutes Wort. Und außerdem, schon der alte Kortschädel sagte mir mal, man müsse gegen Wörter nicht so streng sein und gegen Namen erst recht nicht, da sitze manch einer in einem Glashause.« (284)

Wieder ist der Leser eingeweiht, denn natürlich sitzt auch Adelheid im Glaskasten: Adel - Heide. Jedenfalls aber sind sich die Menschen in solchen Dialogen nicht mehr sicher, wie sie sprechen müssen. Hofmannsthals Ein Brief erscheint nur wenige Jahre später. Die Domina Adelheid in borniert märkischem Adelsstolz und engstirnigem Protestantismus - ihre eigenen Stiftsdamen lassen, wie schon Behrens bemerkt hat ${ }^{36}$ den desolaten Zustand beider Bekenntnisse erkennen - findet, das Wort »beichten« »macht mir jedesmal ein Unbehagen. Es hat solch ausgesprochen katholischen Beigeschmack « (82), und sie bittet kurz darauf Woldemar, wie schon zitiert: »Sage nichts Französisches. Das verdrießt mich immer. Manche sagen jetzt auch Englisches, was mir noch weniger gefällt.« (100) 
Worte haben für sie also eine Wirkung auf den Gemütszustand: Sie machen Unbehagen, verdrießen, gefallen nicht. Wir erfahren dadurch die Weltanschauung der Domina schon aus ihrem eigenen Vokabular und wie sie es zustimmend oder ablehnend verwendet. Nur die Domina kann eine solche Abneigung gegen ausländische Gesprächsbrocken haben, aber der Leser bekommt darüber hinaus den Eindruck, sie verschanze sich mit ihren auf Worte so sensibel reagierenden Nerven vor dem Wandel der Wirklichkeit. Ihre Feinfühligkeit für den Wandel, den sie ablehnt, wird ganz besonders an folgender Stelle sprachlich offenbar, an der sie Woldemar von einem theologischen Gespräch mit dem Rentmeister Fix in ihrem Stift erzählt:

Ach, es handelte sich um das, was uns allen, wie du dir denken kannst, jetzt das Teuerste bedeutet, um den >Wortlaut<. Und denke dir, unser Fix war dagegen. Er mußte wohl denselben Tag was gelesen haben, was ihn abtrünnig gemacht hatte. Personen wie Fix sind sehr bestimmbar. Und kurz und gut, er sagte: das mit dem > Wortlaut<, das ginge nicht länger mehr, die >Werte $<$ wären jetzt anders, und weil die Werte nicht mehr dieselben wären, müßten auch die Worte sich danach richten und müßten gemodelt werden. Er sagte >gemodelt $<$. Aber was er am meisten immer wieder betonte, das waren, die $>$ Werte $<$ und Notwendigkeit der $>$ Umwertung $<$. (98)

Wieder ist das Versteckspiel Fontanes subtil. Er befindet sich mit dem Leser im Bunde gegen seine Romangestalt, denn natürlich weiß der Leser, was Fix gerade gelesen hat: Nietzsche. Könnte es aber sein, daß das Versteckspiel noch weitergeht; daß Fontane in ironischer Verzerrung, weil aus dem denkbar unberufensten Munde, über neue Bauelemente seines Buches spricht? Weil die Werte sich geändert haben, müssen auch die Worte geändert werden?

Es ist kaum eine halbe Seite später, daß Adelheid noch einmal ihre sprachliche Sensibilität zu erkennen gibt, wobei wieder über Worte gesprochen wird. Sie ist im Gespräch mit ihrem Neffen:

»dein Freund, der Hauptmann, [...] hat sie bei Tisch geuzt.« «Geuzt?«

»Du wunderst dich über das Wort, und ich wundre mich selber darüber. Aber daran ist unser guter Fix schuld. Der ist alle Moment mal nach Berlin rüber, und wenn er dann wiederkommt, dann bringt er so was mit, und wiewohl ich's unpassend finde, nehm ich's doch an.« (99)

Wie Adelheid ist auch Dubslaw sich seiner sprachlichen Veraltetheit in der Zeit bewußt, aber anders als seine Schwester benutzt er den ironischen Gebrauch modernen Vokabulars, das er immer als solches beschreibt, zum hu- 
moristischen und gelassenen Hinter-die-Kulissen-Gucken, zur Entlarvung. Er beherrscht beide Sprachen, die seiner eigenen Jugend und der Zeit, in der er noch lebt; die der gräflichen Familie und die Unckes oder des betrunkenen Wählers, den er im Wagen mitnimmt; die seiner Heimat und die der Welt. Er macht lächerlich, was immer lächerlich gemacht zu werden verdient. Er registriert sorgfältig das Verschwinden alter Wörter und das Auftauchen neuer, modischer, denen er dann die vertraute Sprache gegenüberstellt. Der Erzähler sagt etwa von ihm: "Er fuhr noch eine Weile fort, in allerlei Worten und Wendungen, wie sie ihm von alter Zeit geläufig waren [...].« (328) Übrigens tut er das umso häufiger, je näher er dem Tod ist. Auffällig wird es besonders an folgenden Stellen: »Bringe mir lieber einen Roman; früher in meiner Jugend sagte man Schmöker. Ja, damals waren alle Wörter viel besser als jetzt « (314); oder: "weil heutzutage so vieles schwer festzustellen ist, haben sich ja die Menschen auch das angeschafft, was sie 'ne >Enqueter nennen. Keiner kann sich freilich so recht was dabei denken.« (334)

Schon die Zusammenstellung des saloppen »'ne« mit dem Fremdwort »Enquete«zeigt Dubslaws eigenen Abstand dazu. Was er bei solchen sprachlichen Veränderungen immer wieder zu treffen sucht, ist der zunehmende rhetorische Größenwahn des Deutschen Reiches, und er spricht diese Einsicht auch grundsätzlich aus, aber bezeichnenderweise wird auch diese Aussage wie so viele andere in dem Roman, die Prinzipielles beinhalten, wieder ironisiert, denn Dubslaw verfällt dem Fehler, den er gerade moniert:

»Ideosynkrasie«, wiederholte der Alte. »Wenn ich so was höre. Ja, Woldemar, da glaubst du nun wieder wunder was Feines gesagt zu haben. Aber es ist doch bloß ein Wort. Und was bloß ein Wort ist, ist nie was Feines, auch wenn es so aussieht. Dunkle Gefühle, die sind fein. Und so gewiß die Vorstellung, die ich mit dieser lieben Flasche hier verbinde, für mich persönlich was Celestes hat . . Kann man Celestes sagen?«

Lorenzen nickte zustimmend [...]. (69)

Wie sehr auch die junge Generation in den sprachlichen Gepflogenheiten der Zeit, aber zugleich auch immer in ironischem oder kritischem Abstand zu ihnen lebt, zeigen die Gespräche zwischen Woldemar, Rex und Czako. Wenn immer der Leser sie im Dialog miteinander erlebt, reflektieren sie sprachliche Wendungen und ihre Angemessenheit, zum Beispiel an folgender Stelle, an der Czako zu Rex sagt:

»all das kaufte sich meine Mutter. Und an diesem Stereoskopenkasten, ein Wort, das ich damals noch nicht kannte, habe ich meine italienische Kunst gelernt. [...] Was können sie da groß verlangen? Ich habe, wenn sie das Wort gelten lassen wollen, 'ne Panoptikumbildung.« 
Rex lachte. »Nun gleichviel. Also der Graf, der die ältere Komtesse Barby heiratete, hieß Ghiberti. Seiner Ehe fehlten indes durchaus die Himmelstüren - soviel läßt sich mit aller Bestimmtheit sagen. Und deshalb kam es zur Scheidung. Ja, mehr, die charmante Frau (>charmant< ist übrigens ein viel zu plebejes und minderwertiges Wort) hat in ihrer Empörung den Namen Ghiberti wieder abgetan [...].« (107 f.)

Reizvoll ist hier auch, nachdem Czako von italienischer Kunst gesprochen hat, die Anspielung auf Lorenzo Ghibertis berühmte Bronzetüren am Baptisterium des Florentiner Doms, von denen Michelangelo gesagt haben soll, so stelle er sich die Paradiesespforten vor. Noch dichter reihen sich sprachlich reflektierende Einlagerungen in den Dialog, als Waldemar seinen Freunden die Herkunft von Frau Katzler erklärt:

»Um so großartiger, wenn einzelne der hier in Betracht kommenden Damen auf alle diese Vorrechte verzichten und ohne Rücksicht auf Ebenbürtigkeit sich aus reiner Liebe vermählen. Ich sage >vermählen<, weil >sich verheiraten etwas plebeje klingt. Frau Katzler ist eine Ippe-Büchsenstein.«

"Eine Ippe! « sagte Rex. »Nicht zu glauben. Und erwartet wieder. Ich bekenne, daß mich das am meisten schockiert. Die Ausgiebigkeit, ich finde kein anderes Wort, oder richtiger, ich will kein anderes finden, ist doch eigentlich das Bürgerlichste, was es gibt."

»Zugegeben. Und so hat es die Prinzessin wohl auch selber aufgefaßt. Aber das ist gerade das Große an der Sache; ja, so sonderbar es klingt, das Ideale.« $(75 \mathrm{f}$.

Die Dimension sprachlicher Distanzierung ist noch am Ende des Buches auf besonders diskrete Art anwesend. Czakos Liebe zu Melusine, so meint Rex, könne durch eine bloß sprachliche, durch die Änderung seines Namens glücklich enden: »Italienisieren Sie sich und schreiben Sie sich von morgen an Ciacco. Dann sind Sie dem Ghiberti trotz seiner Grafenschaft dicht auf den Hacken.«(383) Dubslaw, der kurz vor seinem Tod seine Einsicht in die Gesetzlichkeit der Welt ausspricht - eine weitere Schlüsselstelle des Buches versucht sich sprachlich vom Tod zu distanzieren: »Ein ewig Gesetzliches vollzieht sich, weiter nichts, und dieser Vollzug, auch wenn er >Tod< heißt, darf uns nicht schrecken.« (372)

$\mathrm{Da}$ Fontane mit Vergnügen den sprachlichen Gepflogenheiten auch die komischen Seiten abgewinnt, versteht sich von selbst. Eine Art der komischen Wirkung besteht darin, daß die Personen mit Worten um den heißen Brei gehen. Aus Antipathie oder gesellschaftlicher Konvention gestatten sie sich nicht, Worte zu gebrauchen, die doch im Gespräch anwesend sind. Jeder 
kennt sie, aber keiner spricht sie aus, was nur bewirkt, daß über sie mehr als nötig geredet wird. So ist es mit den unsäglichen Namen deutscher Weine, besonders dem einen, über den man an der Tafel der Domina diskutiert, Namen, denen "gegenüber uns dann nur noch das Refugium der französischen Aussprache bleibt« (94). Und die Domina, durch die Vorstellung, französisch sprechen zu hören, und durch derlei Wörter in ihrem Patriotismus und ihrer Vornehmheit gekränkt, hebt denn auch prompt die Tafel sofort auf. An einer anderen Stelle ziert sie sich, ein Fremdwort zu gebrauchen, obwohl sie doch darüber reflektiert: »Aber sie wollen ihm ein Rad anschaffen, solches, wie jetzt überall Mode ist. Ich sage Rad, weil ich das fremde Wort, das so verschieden ausgesprochen wird, nicht leiden kann. Manche sagen >ci<, und manche sagen $>$ schi $\ll$ « $(80)$

Die doppelte Ebene der Dialoge im Stechlin, die man als gleichzeitiges Darstellen von erzählender und erzählter Sprache begreifen könnte, vergegenwärtigt in jedem Moment >Zeitgeist` und hält die sozialen, historischen und geographischen Differenzen und Überlagerungen präsent. Die Welt wird nicht nur als Welt im Wandel, als Welt sozialer Schichtungen und als Welt verschiedener Lebensräume vorgeführt, sondern sie wird auch von den Gestalten des Buches so erfahren. Dadurch hat sich Fontanes Sprachbewußtsein im Alter von beinahe 80 Jahren - nicht nur noch einmal gewandelt; der Autor hat sein eigenes neues Sprachverständnis auch an seine Gestalten weitergegeben. Es sei zum Schluß dieser sprachlichen Erörterungen ein kurzes Stück Dialog zitiert, das das Menschliche, das Ironische und das doppeldeutige Spiel mit den Wörtern vereint und Fontanes späte Kunst in ihrer ganzen Liebenswürdigkeit repräsentiert. Die fromme Frau Katzler besucht den alten Stechlin kurz vor seinem Tod und möchte ihm geistliche statt medizinischer Hilfe nahe legen:

»Sogenannte Medikamente sind und bleiben ein armer Notbehelf; alle wahre Hilfe fließt aus dem Wort. Aber freilich, das richtige Wort wird nicht überall gesprochen.«

Dubslav sah etwas unruhig um sich her. Es war ganz klar, daß die Prinzessin gekommen war, seine Seele zu retten. Aber woher kam ihr die Wissenschaft, daß seine Seele dessen bedürftig sei? Das verlohnte sich doch in Erfahrung zu bringen, und so bezwang er sich denn und sagte: »Gewiß, Durchlaucht, das Wort ist die Hauptsache. Das Wort ist das Wunder; es läßt uns lachen und weinen; es erhebt uns und demütigt uns, es macht uns krank und macht uns gesund. Ja, es gibt uns erst das wahre Leben hier und dort.« (328f.) 
Thomas Mann schrieb 1919 in sein Tagebuch: "Las aber mit Entzücken den ganzen $>$ Stechlin . Wundervoll. [...] Für den späten Fontane paßte das Wort $>$ süblim $<. \ll{ }^{37}$ Wer wollte ihm nicht gerne zustimmen!

Aus Fontanes Verarbeitung der kulturanthropologischen Kategorien und der sprachlichen Reflexionen müßte sich eins ablesen lassen: Den Stechlin als Werk langsamen Verfalls seines Verfassers zu betrachten, ist kaum angemessen. Er ist nicht nur Fontanes letzter Roman, sondern nach der Erprobung durch Die Poggenpuhls auch der Gipfel eines neuen Fontaneschen Zeitromans, eines Zeitromans, in dem ohne Romanhandlung im üblichen Sinne durch sprachliche Zusammenhänge die unlösbare Verbindung von Menschen und ihrer Lebenswelt dargestellt wird und privates Leben und Zeitgeist sich durchdringen zu einer künstlerischen Einheit von reichen und enggeknüpften Bezügen.

Es könnte aber nach diesen Bemerkungen scheinen, als ob Fontanes Roman vorwiegend theoretisiert. Gerade dieser Eindruck aber wäre falsch, denn das Bemerkenswerte am Stechlin ist, daß der Autor selbst darin kaum etwas abstrakt-gedanklich Formuliertes sagt. Seine Technik erlaubt es, alle Aspekte der »Idee « des Romans vollkommen einzuschmelzen in die Begegnungen der Menschen. In ihr Verhalten und ihre Gespräche, ja das Theoretische dahinter scheint gar nicht an sich zu existieren; es wird gelebt. Es ist einfach Teil der Alltäglichkeit, und deshalb verzichtet der Autor auf den tragischen Fall, in dem das Individuum mit den moralischen Geboten der Gesellschaft in Konflikt gerät; er wählt eine oberflächlich gesehen banale Geschichte. Die Idee darin ist so unauffällig, daß man geneigt ist, sie zu übersehen. Hat man sie allerdings entdeckt, dann erkennt man, daß alle Lebensäußerungen der Romangestalten davon durchtränkt sind; sie ist allgegenwärtig. Nur so enthüllt sich ihre Bedeutung, ihre Repräsentanz. Nicht das »unerhörte Ereignis« enthält die Ideen des Menschlichen konzentriert in sich, sondern jedes Leben ist nun für Fontane - im Sinne von Wilhelm Diltheys Lebensphilosophie - an sich selbst hermeneutisch.

Wie wohl hätte nach diesen neuen Einsichten Fontanes nächster Roman ausgesehen? 
Anmerkungen

${ }^{1}$ Fritz Martini, Deutsche Literatur im bürgerlichen Realismus, 1848-1898. Stuttgart 1962, S. 782.

${ }^{2}$ Demetz, S. 145.

${ }^{3}$ Vinzenz J. Günther, Das Symbol im erzählerischen Werk Fontanes. Bonn 1967 (=Bonner Arbeiten zur deutschen Literatur, Bd. 16), S. 83.

${ }^{4}$ Helmuth Nürnberger, Theodor Fontane in Selbstzeugnissen und Bilddokumenten. Reinbek 1968 u. ö., (= rowohlts monographien, Bd. 145), S. 138.

${ }^{5}$ Richard Brinkmann, Über die Verbindlichkeit des Unverbindlichen. München 1967, S. 70.

${ }^{6}$ Demetz, S. 145.

${ }^{7}$ Reuter, S. 557.

${ }^{8}$ Demetz, S. 85.

${ }^{9}$ L. Thomas, "Fontanes >Unterm Birnbaum««, in: GLL NS, vol. XXIII, 3 (1970), S. 193-205.

${ }^{10}$ Demetz, S. 195.

${ }^{11}$ Ebd., S. 157.

${ }^{12}$ Ebd., S. 180.

${ }^{13}$ J. P. Stern, Re-Interpretations. London 1964, S. 319.

${ }^{14} \mathrm{M}$. Rychner, »Theodor Fontane >Der Stechlin «, in: Interpretationen III. Deutsche Romane von Grimmelshausen bis Musil. Fischer Tb. 716, S. 218.

${ }^{15}$ Demetz, S. 158.

${ }^{16}$ Ebd., S. 163.

${ }^{17}$ Ebd., S. 162.

${ }^{18}$ Ebd., S. 163.

${ }^{19}$ An Siegmud Schott, 14. 2. 1897 (HB 4.635).

${ }^{20}$ An Georg Friedlaender, 4. 1. 1897 (Briefe Friedlaender, S. 307).

${ }^{21}$ Entwurf an Adolf Hoffmann 1897 (HB 4.649)

${ }^{22}$ München 1963, S. 231.

${ }^{23}$ Ebd., S. 227.

${ }^{24}$ Ebd., S. 237

${ }^{25}$ Ebd., S. 234.

${ }^{26}$ Ebd., S. 238.

${ }^{27}$ Wie Anm. 21.

${ }^{28}$ Martini, a. a. O., S. 779.

${ }^{29}$ Günther, a. a. O., S. 115 , bezieht die Szene fälschlicherweise auf Czako statt Rex, was Fontane aus psychologischen Gründen sicher nicht getan hätte.

${ }^{30}$ Ansätze zu dem hier Gemeinten finden sich höchstens in Mathilde Möhring, wo Mathildes Mutter gelegentlich über die gebildeten Ausdrücke ihrer 
Tochter erschrickt, oder in Unwiederbringlich, wo Ebba sich hin und wieder über die Sprache ihrer Umwelt mokiert.

${ }^{31} \mathrm{~B}$. Rang, "Die deutsche Epik des 20. Jahrhunderts«, in: Deutsche Literatur im 20. Jahrhundert, hg. von Otto Mann und Wolfgang Rothe. München ${ }^{5} 1967$, S. 1.95.

${ }^{32}$ Erich Rothacker, Probleme der Kulturanthropologie. Bonn ${ }^{2} 1948$. Rothacker verwendet als solche kulturanthroplogischen Begriffspaare, in deren Spannungsfeld sich kulturelles Leben abspielt, etwa Universalismus - Partikularismus, Spiritualismus - Sensualismus, Stadt - Land u. a.

${ }^{33}$ Demetz, S. 157.

${ }^{34}$ Andeutende Hinweise gibt es, soweit ich sehe, nur bei Reuter, S. $855 \mathrm{ff}$.

${ }^{35} \mathrm{Vgl}$. dazu Herman Meyer, Das Zitat in der Erzählkunst. Zur Geschichte und Poetik des europäischen Romans. Stuttgart 1961, S. 155 ff.

${ }^{36}$ E. Berens, Theodor Fontanes Roman »Der Stechlin «. Marburg 1929, S. 31.

${ }^{37}$ Tagebücher 1918-1921, hg. von Peter de Mendelssohn. Frankfurt am Main 1979, S. 309 (24. und 25. 10.). 